\title{
Alterstice
}

Revue internationale de la recherche interculturelle

International Journal of Intercultural Research

Revista International de la Investigacion Intercultural

\section{Dispositif de télécollaboration pour des étudiants universitaires : littérature migrante, autobiographie migratoire et échanges interculturels en ligne}

\section{Myra Deraîche et Christèle Maizonniaux}

Volume 8, numéro 1, 2018

Jumelage interculturel et pédagogie universitaire

URI : https://id.erudit.org/iderudit/1052610ar

DOI : https://doi.org/10.7202/1052610ar

Aller au sommaire du numéro

Éditeur(s)

Alterstice

ISSN

1923-919X (numérique)

Découvrir la revue

Citer cet article

Deraîche, M. \& Maizonniaux, C. (2018). Dispositif de télécollaboration pour des étudiants universitaires : littérature migrante, autobiographie migratoire et échanges interculturels en ligne. Alterstice, 8(1), 83-94.

https://doi.org/10.7202/1052610ar

\section{Résumé de l'article}

Deux enseignantes-chercheuses développent un dispositif de télécollaboration (d'échange interculturel en ligne) réunissant des étudiants de français langue seconde à Montréal (Canada) et des étudiants de français langue étrangère à Adélaïde (Australie). Ce dispositif repose sur une approche innovante, exploitant des textes de littérature migrante et des récits autobiographiques pour la lecture-écriture. Afin de déterminer s'il comble les besoins des deux groupes, s'il favorise l'engagement des apprenants dans les échanges écrits sur la plateforme et s'il aboutit à la production d'écrits autobiographiques riches et pertinents après les lectures partagées et les échanges entre pairs, on a comparé les éléments clés du dispositif aux résultats d'une analyse de données. On peut en conclure que proposer des activités communes s'avère pertinent pour l'expression de soi et l'échange interculturel. 


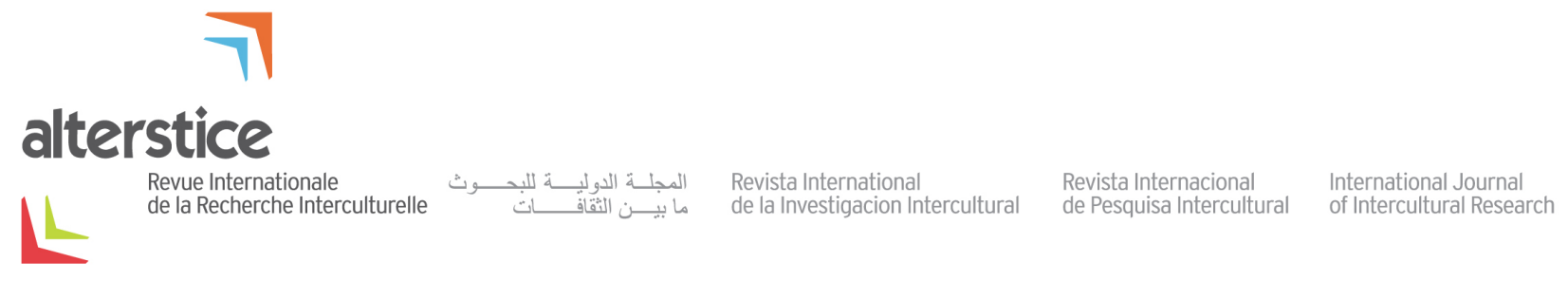

ARTICLE THÉMATIQUE

\section{Dispositif de télécollaboration pour des étudiants universitaires : littérature migrante, autobiographie migratoire et échanges interculturels en ligne}

Myra Deraîche ${ }^{1}$ et Christèle Maizonniaux ${ }^{2}$

\section{Résumé}

Deux enseignantes-chercheuses développent un dispositif de télécollaboration (d'échange interculturel en ligne) réunissant des étudiants de français langue seconde à Montréal (Canada) et des étudiants de français langue étrangère à Adélaïde (Australie). Ce dispositif repose sur une approche innovante, exploitant des textes de littérature migrante et des récits autobiographiques pour la lecture-écriture. Afin de déterminer s'il comble les besoins des deux groupes, s'il favorise l'engagement des apprenants dans les échanges écrits sur la plateforme et s'il aboutit à la production d'écrits autobiographiques riches et pertinents après les lectures partagées et les échanges entre pairs, on a comparé les éléments clés du dispositif aux résultats d'une analyse de données. On peut en conclure que proposer des activités communes s'avère pertinent pour l'expression de soi et l'échange interculturel.

\section{Rattachement des auteures}

${ }^{1}$ Université du Québec à Montréal, Montréal, Canada; ${ }^{2}$ Flinders University, Adélaïde, Australie

\section{Correspondance}

deraiche.myra@uqam.ca

\section{Mots clés}

télécollaboration, littérature migrante, autobiographie, français langue seconde, français langue étrangère

\section{Pour citer cet article}

Deraîche, M. et Maizonniaux, C. (2018). Dispositif de télécollaboration pour des étudiants universitaires : littérature migrante, autobiographie migratoire et échanges interculturels en ligne. Alterstice, 8(1), 83-94. 


\section{Introduction}

Aujourd'hui, les communications en ligne sont massivement transnationales et interculturelles. Un peu partout dans le monde, des individus de diverses langues et cultures échangent sur le web. Ce dialogue virtuel mondial s'accompagne malheureusement d'une montée des discours haineux en ligne (ECRI, 2016). Dans cette perspective, le Conseil de l'Europe lance un mouvement contre la haine (Conseil de l'Europe, 2014) et l'UNESCO publie un ouvrage portant sur la lutte au discours haineux en ligne (Gagliardone, Gal, Alves et Martinez, 2015). Le Conseil de l'Europe (2014) souligne qu'il est important d'éduquer la jeunesse sur les discours en ligne à caractère raciste et xénophobe pour assurer une meilleure cohésion sociale. Le but est de former des citoyens capables de mener des échanges interculturels sur le web tout en faisant preuve de respect face à la différence (Helm, 2014). On peut s'interroger sur le rôle que peuvent jouer les institutions universitaires à cet égard.

L'une des voies possibles pour la pédagogie universitaire et la formation interculturelle est la télécollaboration. Elle consiste en des échanges en ligne entre individus de diverses langues et cultures qui apprennent une langue. Plus spécifiquement, la " télécollaboration et l'échange virtuel sont des termes utilisés pour désigner l'engagement de groupes d'apprenants dans des interactions interculturelles en ligne et des projets de collaboration avec des partenaires d'autres contextes ou d'autres lieux géographiques. Ces interactions et collaborations font partie intégrante de leur programme éducatif ॥ (O’Dowd, 2018, p. 1, traduction libre). II faut préciser que, depuis près de 25 ans, la télécollaboration suscite un vif intérêt dans les écrits scientifiques (Lewis et O’Dowd, 2016). Ces échanges se multiplient un peu partout dans le monde, bien qu'ils soient plus répandus en Europe et en Amérique du Nord (Cziko, 2004; Lewis et O’Dowd, 2016). Or, selon les chercheurs de la plateforme Unicollaboration (2014), la télécollaboration répond aux besoins des universités de l'avenir. Cette formule pédagogique à faible coût permet aux institutions de viser des habiletés du $21^{\mathrm{e}}$ siècle avec le développement des langues étrangères, de la sensibilité interculturelle et de la pensée critique (Unicollaboration, 2014). Cependant, dans les programmes d'échange en ligne d'étudiants volontaires, il est reconnu que ce sont les candidats qui ont déjà des expériences interculturelles qui s'y aventurent (Helm, 2014). Les universités ont précisément le pouvoir de rejoindre les étudiants récalcitrants, ceux qui sont peu portés vers l'échange interculturel, dans le cadre de leurs activités obligatoires et de leurs programmes de formation (Helm, 2014). Malgré l'intérêt suscité par la télécollaboration, il reste tout un travail de recherche à effectuer pour mieux cerner les modalités qui facilitent l'apprentissage de la langue et des compétences de communication interculturelle (Chun, 2015).

Dans cet article, il est question d'un projet de recherche visant le développement d'un dispositif de télécollaboration. Deux enseignantes-chercheuses ont conçu un dispositif pour leurs étudiants, qui a consisté en des activités de lecture, d'écriture et d'échange pour l'apprentissage du français sur une plateforme web. La dimension interculturelle du projet réside dans (1) l'échange à distance entre apprenants de diverses cultures à partir de la lecture de textes spécifiques, (2) l'échange à distance sur sa vie personnelle avec un interlocuteur d'une autre culture, (3) les échanges à l'intérieur des classes entre apprenants d'origines diverses, que ce soit sur le site de Montréal ou d'Adélaïde. Il faut préciser que les étudiants provenaient de deux universités : des adultes immigrants inscrits dans une université francophone, l'Université du Québec à Montréal (UQAM), et de jeunes étudiants d'une université australienne anglophone, Flinders University à Adélaïde (Australie).

L'article vise à comparer les éléments clés du dispositif et les résultats de l'analyse afin de déterminer si l'approche envisagée permet de répondre aux objectifs fixés par les enseignantes-chercheuses. Dans cette optique, nous exposons d'abord les éléments de contexte cruciaux ayant présidé au développement d'un dispositif spécifique, puis nous présentons les assises théoriques sur lesquelles repose le dispositif, les objectifs de recherche et la méthodologie. Ces divers éléments sont par la suite intégrés à une synthèse des résultats issus de l'analyse des données pour identifier, entre autres, les points forts et les points faibles du dispositif. 


\section{Élaborer un dispositif en tenant compte des contextes et besoins d'apprentissage}

\section{Besoins d'apprentissage}

La télécollaboration a réuni des étudiants aux besoins sociolinguistiques différents. D'une part, à l'UQAM, les apprenants constituaient une population migrante désireuse d'apprendre le français langue seconde ${ }^{1}$ pour s'intégrer professionnellement et culturellement. II s'agit d'une population qui manque de contacts réguliers significatifs avec la majorité francophone, même si elle vit dans une province francophone du Canada (Carignan, Deraîche et Guillot, 2015). D'autre part, à Flinders, les étudiants des cours de français évoluaient dans un univers anglophone où se présentent peu d'occasions de pratiquer la langue cible. Ces apprenants désiraient acquérir le français langue étrangère pour enrichir leur profil universitaire et professionnel (Maizonniaux, Deraîche et Saffari, 2017) et éventuellement effectuer un séjour linguistique dans un pays francophone. Dans cet esprit, il s'agissait d'élaborer un dispositif de télécollaboration apte à ce que ces deux groupes d'apprenants puissent pratiquer et développer ensemble leurs compétences en langue française.

Dans les deux contextes universitaires, les enseignantes-chercheuses visaient le développement de la lectureécriture (Reuter, 1994). Les apprenants immigrants en langue seconde ont besoin de développer des compétences de niveau avancé en français écrit pour pouvoir travailler, étudier et s'intégrer à la société (Adami, 2008). De ce fait, il importe que les personnes immigrantes qui désirent prendre une part active dans la société développent leurs compétences en lecture-écriture. Quant au groupe de français langue étrangère en Australie, les exigences de l'institution en matière d'internationalisation du parcours ont amené l'enseignante à exploiter la littérature tout en maintenant les objectifs linguistiques, littéraires et culturels qui sont ceux de l'enseignement du français langue étrangère en contexte universitaire à un niveau de compétence avancé. Le défi était de concevoir un dispositif de télécollaboration visant à la fois la lecture-écriture en français et la littérature francophone, puis de répondre aux objectifs fixés par les deux enseignantes pour leurs groupes respectifs.

\section{Dispositif de télécollaboration}

Les enseignantes-chercheuses ont voulu développer un dispositif de télécollaboration favorisant en premier lieu l'apprentissage des étudiants des deux universités. Or il ne suffit pas de mettre des apprenants en contact par Internet pour qu'ils acquièrent la langue et la culture ou pour que le dispositif soit adéquat. Comme le soulignent Ollivier et Puren (2014), "le fait de recourir aux outils du Web 2.0 à des fins éducatives ne débouche pas nécessairement sur une pédagogie innovante »(p. 61). Dans les écrits scientifiques et pédagogiques sur la télécollaboration, la question de la conception et de l'efficacité du dispositif préoccupe les chercheurs (Appel et Mullen, 2000; Cziko, 2004; O'Dowd et Waire, 2009). D'après O'Dowd (2006), l'efficacité du dispositif pour l'atteinte d'objectifs langagiers, grammaticaux, interculturels et éducatifs constitue l'un des quatre grands axes de réflexion de la recherche sur la télécollaboration.

Si la conception des dispositifs de télécollaboration est au cœur de la recherche, c'est qu'elle pourrait faire toute la différence sur l'apprentissage. Ainsi, dans leur revue systémique des recherches empiriques sur l'impact de la télécollaboration sur l'apprentissage des langues, Lewis et O'Dowd (2016) affirment que les tâches doivent être savamment construites pour produire un effet : « de [notre] point de vue, des données probantes existent dans les écrits scientifiques qui suggèrent que la télécollaboration peut contribuer au développement d'une langue seconde, particulièrement quand les tâches sont consciencieusement conçues pour encourager l'attention sur la forme et quand les échanges en ligne sont combinés à des réflexions et activités hors ligne » (p. 64, traduction libre). Les experts qui gèrent la plateforme mondiale de télécollaboration universitaire Unicollaboration sont du même avis. Ils soutiennent que la télécollaboration est bénéfique, mais qu'il existe de réels besoins de recherche, de développement et d'évaluation dans ce domaine (Unicollaboration, 2014).

\footnotetext{
${ }^{1}$ En didactique des langues, on distingue les concepts de langue seconde et de langue étrangère. La langue seconde correspond à une langue supplémentaire apprise (supplémentaire à la langue maternelle) et cette langue visée a un statut politique officiel dans le pays (par exemple le français est une des deux langues officielles au Canada) (Legendre, 2005). La langue étrangère est une " autre langue que la langue maternelle [qui] ne possède pas de statut politique officiel dans le pays où elle est enseignée » (Legendre, 2005, p. 828).
} 
Il existe par ailleurs un manque de documentation sur la télécollaboration impliquant une population migrante. Dans les écrits scientifiques et pédagogiques sur les échanges interculturels en ligne, les projets incluent surtout des apprenants de langue étrangère ou de futurs enseignants de langue (Akiyama et Cunningham, 2018). Dans un numéro spécial de la revue Les langues modernes consacré à la télécollaboration, un seul article mentionne des personnes immigrantes apprenant une langue seconde (Maizonniaux, Deraîche et Saffari, 2017). Dans sa synthèse sur la télécollaboration, O'Dowd (2018) ouvre le domaine aux projets interculturels multidisciplinaires, mais sans mentionner de modalités télécollaboratives spécifiques à des populations marginalisées comme les étudiants issus de l'immigration. Or les personnes immigrantes peuvent profiter des échanges interculturels en ligne non seulement pour apprendre à maîtriser les nouvelles technologies en langue seconde, mais aussi pour discuter et amener une conscientisation interculturelle (Amireault et Deraîche, 2015). II serait souhaitable d'enrichir le domaine de recherche de la télécollaboration relativement à ces apprenants, ce qui est visé dans le présent projet.

Dans une première phase, les deux enseignantes-chercheuses ont déterminé les besoins d'apprentissage particuliers en lien avec les contextes universitaires des deux groupes d'apprenants. De plus, en examinant les écrits scientifiques sur la télécollaboration, il a semblé clair que la conception d'un dispositif efficace s'avérait un problème de recherche en soi. Les écrits scientifiques dans le domaine n'ont pas encore réussi à dégager un modèle pédagogique efficient de télécollaboration et la recherche doit se pencher sur ce problème. En outre, les études abordent peu le cas spécifique des personnes immigrantes qui apprennent une langue dans le cadre de la télécollaboration. Dans la section suivante, il est question des assises théoriques qui sous-tendent la conception du dispositif.

\section{Assises théoriques pour le dispositif}

\section{Lire la littérature migrante en contexte scolaire ou universitaire}

La littérature migrante se définit comme " l'ensemble des textes littéraires qui abordent le sujet de l'immigration, qu'ils soient écrits par un auteur issu de l'immigration ou non " (De Croix et Ledur, 2014, p. 177). Même si dans les espaces francophones on traite depuis longtemps de la migration, bien avant les années 1980 où l'étiquette de littérature migrante est apparue au Québec, il s'agit surtout d'une reconnaissance de la pluralité culturelle dans la littérature (Bélair, 2010).

Dans les pays francophones, la littérature migrante occupe aujourd'hui une place de choix dans les publications en librairie. Notamment, le recueil de Barbizet-Namer (2009) en France présente 50 auteurs qui racontent l'immigration. Des portraits d'immigrants sont également livrés dans l'ouvrage québécois Voix migrantes (Kunigis et Marcotte, 2014). De plus, cette littérature est associée à des auteurs reconnus internationalement comme Kim Thuy, Dany Laferrière ou Nancy Huston.

En didactique du français, certains écrits abordent ce mouvement littéraire comme un corpus pertinent pour l'enseignement de la langue et de la culture. Lebrun et Collès (2007) prétendent que « [par] la littérature migrante, l'élève développera des connaissances, tant littéraires que sociales au sens large, sur la diversité culturelle de la société québécoise » (p. 16). D’ailleurs, dernièrement, un manuel de français langue seconde intitulé Voix croisées a été publié (Jobin et Tamiozzo, 2016). II réunit et exploite, entre autres, des œuvres de grands artistes d'origines diverses publiant des textes littéraires en langue française. Ces exemples mettent en avant la légitimité de cette littérature en contexte scolaire et soulignent qu'elle peut constituer un outil précieux pour aborder la culture et la diversité. Dans cette perspective, il a semblé opportun d'intégrer dans le dispositif de télécollaboration une série de textes de littérature migrante, des textes d'auteurs publiés en France ou au Québec, pour développer la lectureécriture et pour nourrir les échanges interculturels.

\section{Écrire son autobiographie}

En éducation, la biographie et l'autobiographie sont de nouvelles voies de recherche et offrent des possibilités didactiques. Par exemple, le Conseil de l'Europe a mis en place un site internet intitulé l'Autobiographie des rencontres interculturelles expliquant que l'autobiographie " est un outil conçu pour encourager les utilisateurs à réfléchir sur les rencontres interculturelles qu'ils ont faites ». En outre, aborder la biographie et le parcours 
langagier permet de "faire prendre conscience aux enseignants de la réalité plurilingue » (Molinié, 2011, p. 147). Par ailleurs, avec ce que Molinié (2011) nomme les « approches biographiques des parcours et des mobilités plurilingues ", il est possible, dans la classe de langue, de mettre en discours le parcours du sujet plurilingue, en migration ou descendant de migrants.

En ce qui concerne la lecture et la production de textes autobiographiques en classe de langue, peu de recherches ont été effectuées dans ce domaine. Certaines recherches mettent en avant l'engagement important des apprenants migrants impliqués dans l'écriture de leurs propres textes autobiographiques (Clifford et Kalyanpur, 2011; Vatz Laaroussi, Armand, Kanouté, Rachedi, Steinbach, Stoica et Rousseau, 2010). Les recherches dans le domaine de l'écriture autobiographique en langue première sont également utiles pour notre champ. À l'instar de Cardell et Douglas (2016), on peut dire qu'écrire sa vie contribue à développer des compétences utiles dans d'autres domaines de la vie. En ce qui concerne les dispositifs propices à l'écriture autobiographique, les recherches conduites en langue première dans le secondaire s'avèrent instructives. Recourir à des textes autobiographiques pour susciter la production de textes personnels est une pratique qui fait sens et c'est «ce qui préserve le mieux la liberté de l'élève » (Lejeune, 2001, p. 20). Roche (2001) préconise également d' « écrire avec les auteurs " (p. 49).

\section{Concevoir un dispositif de télécollaboration : quelques éléments clés}

Comme nous l'avons mentionné précédemment, il ne semble pas exister dans les écrits scientifiques ou pédagogiques de modèle pour la télécollaboration. En consultant ces écrits, il est tout de même possible de faire ressortir quelques éléments clés.

Dans son texte sur le rôle de l'enseignant et les enjeux pédagogiques en télécollaboration, Müller-Hartman (2007) présente les phases générales d'un projet de télécollaboration. La télécollaboration comprendrait ainsi trois phases : 1) l'établissement du contact, 2) l'établissement du dialogue et 3) la réflexion critique. L'établissement du dialogue vise à amorcer la communication entre les participants à la télécollaboration. Cette première phase est importante pour que les participants apprennent à se connaître et commencent à s'impliquer dans les échanges. L'établissement du contact peut s'effectuer par l'entremise de lettres de présentation, de photos, de vidéos ou de tout autre élément qui évoque les cultures des partenaires. La deuxième phase, l'établissement du dialogue, consiste à enclencher les échanges bidirectionnels et à favoriser davantage l'interaction. Le dialogue peut démarrer en parlant d'une lecture par exemple. À cette étape, il s'agit de dialoguer le plus possible pour en apprendre le maximum l'un sur l'autre. Enfin, la télécollaboration est conçue de façon à susciter la réflexion à la fin du projet et à amener les participants à user de leur esprit critique. Il pourrait être question d'une discussion sur un enjeu qui a été soulevé lors de la télécollaboration ou sur un élément mal compris. Ici, dans l’optique des échanges interculturels, il s'agit d'aborder les points de rupture et d'envisager des solutions pour de meilleures communications interculturelles dans le futur. Ces phases générales d'une télécollaboration ont inspiré les deux enseignantes-chercheuses dans la préparation de leur dispositif.

Quant aux tâches à intégrer dans une télécollaboration, O’Dowd et Waire (2009) décrivent des catégories générales aptes à guider les enseignants qui montent un projet. Après avoir fait une revue systématique des recherches sur la télécollaboration et avoir réalisé deux études de cas, ces chercheurs ont défini trois grandes familles de tâches : 1) les échanges d'informations, 2) les tâches de comparaison et d'analyse et 3) les tâches créatives de collaboration et de production. Les échanges d'informations incluent par exemple l'envoi de données sur son pays, sa culture, sa langue ou sur d'autres thèmes. Ces tâches sont moins complexes en termes d'activité cognitive et peuvent être plus proches du monologue successif. La deuxième catégorie renvoie aux tâches où les apprenants comparent des textes, des vidéos, des images et analysent les différences, les similarités puis en discutent ensemble. Ici, l'étudiant est amené à s'exprimer davantage et à expliquer divers éléments culturels ou linguistiques. Dans un dernier temps, on inclut toutes les tâches qui demandent aux partenaires de travailler ensemble, de construire ou de créer un produit en toute collaboration. Ce type de tâche demande une bonne dose de communication et de compréhension. C'est ici que la véritable télécollaboration se manifeste, puisque les apprenants doivent bien s'entendre et travailler d'un commun accord. C'est en considérant ces catégories d'O'Dowd et Waire (2009) que les deux enseignantes-chercheuses ont conçu le dispositif de télécollaboration présenté ici. 


\section{Objectifs de recherche}

Pour évaluer le dispositif de télécollaboration inspiré des assises théoriques évoquées dans la section précédente, les enseignantes-chercheuses ont procédé à la mise en place d'un projet de recherche dont les objectifs étaient les suivants :

- Évaluer la pertinence du dispositif. Autrement dit, évaluer globalement et en détail comment le dispositif répond aux besoins d'apprentissage des étudiants des deux universités dans leur contexte respectif;

- Observer les indicateurs d'engagement des étudiants des deux universités dans le cadre du dispositif conçu;

- Observer comment les textes autobiographiques produits témoignent d'appropriations sur les plans linguistiques et littéraires par rapport aux textes migratoires proposés en lecture;

- Observer dans quelle mesure les textes proposés suscitent un dialogue interculturel au sein des paires et retracent des expériences interculturelles.

\section{Méthodologie}

\section{Approche méthodologique choisie}

Le projet de recherche s'inscrit dans ce qu'on appelle en éducation une recherche de type appliquée. Comme l'explique Van der Maren (2010), "la recherche appliquée tente de résoudre des problèmes en proposant des pistes de solutions " (p. 73). Pour lui, ce type de recherche comporte une analyse de besoin initiale, la mise au point d'une action éducative et une phase finale d'évaluation. Dans une première phase, les enseignanteschercheuses ont cerné les besoins d'apprentissage et les contextes universitaires. À partir des assises théoriques, elles ont alors conçu un dispositif de télécollaboration, qui s'avère l'action visée. Les objectifs de la recherche correspondent à la phase d'évaluation de cette action.

Cette recherche relève du paradigme qualitatif. II faut spécifier que les recherches en télécollaboration sont largement dominées par les approches qualitatives: les analyses se doivent d'être hautement contextualisées, puisque chaque dispositif de télécollaboration est produit dans un contexte particulier et que chaque participant s'exprime dans une situation unique (Chun, 2015). De plus, comme les échanges sont transnationaux et qu'ils peuvent se produire dans les classes comme à l'extérieur de celles-ci, il s'avère ardu de contrôler toutes les variables qui influencent ces échanges (Muller-Hartmann, 2000). Dans cette optique, une approche qualitative est adoptée, d'autant plus que les données sont des textes issus de diverses méthodes de recherche (par questionnaire et par analyse de discours) et recueillis en multimodalité (textes en format Web et textes en format Word) (Chun, 2015).

\section{Dispositif conçu et contraintes}

Avant de préciser les résultats de la recherche en mettant ceux-ci en relation avec les éléments clés du dispositif de télécollaboration, il est nécessaire de procéder à la description du dispositif. Cette présentation souligne aussi les contraintes identifiées lors de la phase de conception.

Le dispositif de télécollaboration a couvert une durée de 7 semaines. II comprenait un corpus de textes à lire et d'activités à compléter chaque semaine. Ce corpus et ces activités étaient centrés sur la littérature migrante et l'autobiographie. De plus, des activités étaient proposées en lien avec la lecture des textes. En résumé, les activités ont suivi cette typologie : 1) questions de compréhension sur un texte à lire, 2) activités d'écriture à la manière de l'auteur lu et 3) expression d'un jugement esthétique sur les extraits lus (discussions autour d'un passage jugé important par les apprenants, par exemple). Par ailleurs, les étudiants ont été invités à échanger directement entre eux sur la plateforme (commentaires sur une photographie, écriture d'un petit texte de présentation de soi, écriture de textes autobiographiques courts à partir d'un élément déclencheur).

D'un point de vue plus technique, l'outil technologique retenu a été la plateforme Moodle. II s'agit d'une plateforme d'apprentissage employée internationalement dans les collèges et les universités. Les enseignants 
peuvent y concevoir des modules d'apprentissage pour les étudiants. Ces derniers peuvent y interagir entre eux et avec les enseignants. La plateforme a été choisie à la fois parce qu'elle concordait avec le dispositif télécollaboratif conçu et parce qu'elle est gérée par une institution d'enseignement qui peut assurer la confidentialité des données. Dans le cadre du projet, c'est la plateforme Moodle de I'UQAM qui a été employée, et seules les deux enseignantes-chercheuses ont accès aux données de recherche.

Diverses contraintes ont été identifiées lors de la conception du dispositif de télécollaboration. En ce qui concerne les contraintes de temps et de calendrier, il faut souligner le problème posé par la superposition seulement partielle des semestres de cours dans les deux pays et le nombre limité de semaines pouvant donner lieu à des échanges. De ce fait, le dispositif n'a pu intégrer un nombre important de textes de littérature migrante (six seulement). En outre, les étudiants du groupe australien étaient en congé deux semaines alors que leurs partenaires au Québec continuaient d'avoir cours. Une autre contrainte est liée au nombre déséquilibré de participants d'un côté et de l'autre. Compte tenu du petit nombre d'étudiants inscrits dans les niveaux avancés côté australien, l'enseignante-chercheuse québécoise n'a pu faire participer l'intégralité de ses étudiants. En ce qui concerne les contraintes institutionnelles, elles sont essentiellement liées à l'évaluation, puisque chaque apprenant devait recevoir en fin de semestre une note. Les modalités d'évaluation étant distinctes dans les deux universités, certaines activités ont été exclues du dispositif parce qu'elles ne correspondaient pas aux exigences d'évaluation respectives.

Avant sa mise en place effective, le dispositif a été testé. Les six textes sélectionnés par les enseignantes (voir Annexe A) ainsi que les activités ont été expérimentés de part et d'autre. Lors de la conception du dispositif, les points clés abordés ont porté sur les aspects pratiques (questions de calendrier, nombre d'étudiants) comme sur les aspects pédagogiques (profil des étudiants, besoins des étudiants, objectifs pédagogiques). Enfin a été déposée une demande d'approbation éthique pour la recherche auprès des comités d'éthique des deux universités. Ces autorisations ont été obtenues avant le démarrage de la télécollaboration.

\section{Collecte des données}

La collecte de données a lieu au semestre d'automne 2015 (septembre et octobre 2015). Les deux enseignanteschercheuses ont recruté des volontaires dans les deux groupes d'étudiants de français (un groupe à l'UQAM et un groupe à Flinders). Ces volontaires étaient au nombre de $22: 11$ d'entre eux étaient apprenants de français langue seconde à l'UQAM (3 hommes et 8 femmes), les 11 autres étant apprenants de français langue étrangère à Flinders University (1 homme et 10 femmes). Chaque participant a été jumelé à un participant de l'autre université de sorte que onze dyades ont pris place sur la plateforme de télécollaboration.

Plusieurs types de données ont été collectées. D’abord, il s'agissait de recueillir les productions écrites finales des participants, c'est-à-dire leurs textes autobiographiques. Ensuite, un questionnaire pré-expérimentation et un questionnaire post-expérimentation à développement court (réponse en quelques lignes) ont été présentés à tous les participants. Le questionnaire pré-expérimentation, à douze questions, a été soumis avant le début des échanges en télécollaboration et le questionnaire post-expérimentation, à huit questions, a été proposé à la suite de la télécollaboration. En troisième lieu, soulignons qu'un compte-rendu post-expérimentation à rédiger en ligne (200 mots) a été demandé à tous. Ce compte-rendu visait à synthétiser l'expérience en faisant ressortir les aspects négatifs et positifs. Enfin ont été colligées les réponses hebdomadaires aux activités proposées en ligne et, le cas échéant, les échanges écrits entre les participants pour ces activités. Ces données ont servi à observer la portée interculturelle des échanges.

\section{Analyse des données}

Les enseignantes-chercheuses ont eu recours à l'analyse de contenu selon Patton (2002) pour l'analyse des questionnaires pré- et post-expérimentation ainsi que pour l'analyse du compte-rendu post-expérimentation. Pour ce qui est de l'analyse des productions écrites, le cadre utilisé est celui de Maizonniaux (2018), qui permet de relever diverses traces d'engagement des apprenants dans la lecture et l'écriture. 
Dans une phase finale, les résultats de l'analyse des textes écrits (réponses aux activités proposées en ligne et productions autobiographiques finales) ont été croisés avec les dires des apprenants (analyse des réponses aux questionnaires et du compte-rendu post-expérimentation). Il s'agissait dans cette phase finale de voir si les dires des apprenants corroboraient ou non les résultats des analyses de leurs écrits.

\section{Résultats et discussion}

\section{Évaluation globale et détaillée du dispositif}

L'évaluation globale du dispositif amène à remarquer des différences de perception entre les participants des deux groupes. Les réponses au questionnaire final apportent des éléments pertinents à ce sujet. En effet, ce questionnaire interrogeait les participants sur les points forts et faibles du dispositif. L'analyse de ces réponses montre que les étudiants d'UQAM ont cité comme aspects positifs à la fois des résultats sur le plan académique (amélioration de l'écrit) et sur le plan de la collaboration alors que, pour les étudiants de Flinders, les forces du dispositif se situaient essentiellement dans le collaboratif, dans la communication, dans les échanges écrits par l'entremise de la plateforme Moodle. L'importance accordée à l'écrit par les étudiants de I'UQAM pourrait s'expliquer par l'urgence ressentie par ce groupe d'améliorer leurs compétences à l'écrit au regard de leur projet professionnel et d'intégration dans leur société d'accueil. L'importance accordée par les étudiants de Flinders aux échanges et à la communication pourrait s'expliquer par l'isolement qui est le leur si l'on considère leur faible exposition au français parlé en dehors du temps de classe.

En examinant en détail les points forts cités, il est à remarquer que ceux-ci touchent à l'amélioration des compétences linguistiques et des compétences écrites pour quatre apprenants (enrichir son vocabulaire (U4 ${ }^{2}$ ), améliorer et pratiquer l'écrit (U3, U7 et U11). Sont également cités comme aspects appréciés les textes proposés (F2), les activités (U2), les échanges avec les partenaires de l'autre université (F2, F5, F7, U2, U3, U5 et U8) et enfin le recours à la technologie (U5).

Le compte-rendu post-expérimentation complémente de manière intéressante les réponses obtenues dans le questionnaire final. Nombreux sont ceux qui notent l'opportunité qui leur a été donnée d'échanger de manière authentique - de pratiquer la langue française - sur divers sujets. "Ce fut des échanges brefs, mais très constructifs, par exemple nous avons discuté sur l'utilisation de la technologie en éducation et son importance au niveau de la classe primaire, secondaire et le concept relationnel. » (U8) «Ce jumelage pourrait être plus actif et plus intéressant si tous les participants faisaient ses [sic] devoirs à l'heure. » (U4) « au début [ça] c'était [sic] bien passé, mais dans les dernières activités, j'ai remarqué un petit relâchement de la part de ma jumelle » et l'étudiante d'ajouter qu'elle regrette la non-diversification des thèmes (U2), idée reprise par U1. U9 trouve l'expérience négative au vu de ses attentes, des lectures proposées, de la quantité de travail demandée et aussi en raison de l'abandon de sa jumelle au milieu du projet. II en va de même pour U6. U7 se déclare satisfait. La dyade 11 est la seule à échanger à cette ultime étape du projet. Les témoignages de cette dyade sont positifs et mettent en exergue, pour la participante d'UQAM, que "les sujets agitaient ses émotions ", mais que celle-ci était contente de " partager ses pensées » avec sa partenaire. L'échange final se termine par l'expression réciproque de vœux à la partenaire, non pas de manière polie, mais avec émotion, ainsi que par l'échange de leurs adresses courriel respectives.

\section{Indicateurs d'engagement dans la télécollaboration}

En scrutant les traces écrites des apprenants, les chercheuses constatent que, dans les échanges sur la plateforme, les étudiants sont parvenus à parler d'eux-mêmes, de leur vécu, de leurs expériences. Bien souvent ils ne se sont pas contentés de rédiger une ou deux phrases, mais ont écrit des paragraphes d'au moins dix lignes, communiquant ainsi de nombreuses informations. Par ailleurs, l'analyse fait ressortir la richesse de leurs écrits, la

\footnotetext{
${ }^{2}$ Les participants au projet de recherche sont désignés par un code alphanumérique. Les lettres sont associées aux institutions universitaires. Les abréviations U1, U2, U3 servent ainsi à désigner des étudiants d'UQAM, les abréviations F1, F2, F3, pour des étudiants de Flinders University. Les numéros employés font référence aux dyades formées par les étudiants des 2 universités. Dans cet esprit, U1-F1 forment la première dyade, U2-F2 la seconde, etc.
} 
finesse de leurs remarques par rapport aux questions de migration et aux questions connexes, et ce, chez les participants des deux groupes. Par contre, il est à noter la distanciation des étudiants d'UQAM. Ceux-ci étaient plus âgés et avaient exercé dans leur pays d'origine dans des domaines comme la médecine, la pharmacie, l'ingénierie. La maturité et le recul de ces étudiants, souvent pères et mères de famille, étaient susceptibles de constituer un décalage par rapport au profil de la majorité des étudiants de Flinders, âgés d'une vingtaine d'années. Or il ne semble pas que ce décalage ait posé problème. Au contraire, dans les textes des étudiants australiens, il ressort une certaine curiosité - et une certaine admiration - vis-à-vis leurs partenaires désireux de s'intégrer au Québec.

En ce qui concerne le nombre d'interactions écrites (on qualifie d'interactions la rédaction successive - et en alternance - de textes se répondant), elles s'avèrent plus fréquentes dans certaines dyades que dans d'autres et la qualité de ces interactions varie. En somme, l'ensemble des dyades a bien fonctionné à l'exception des dyades 3,6 et 9 . Dans ces trois cas, l'un des participants ne s'est pas investi régulièrement, que ce soit au début, à la fin ou tout au long de la télécollaboration. Cependant, une tendance se dessine: dans pratiquement tous les binômes, les participants n'utilisent pas de manière systématique la fonction « répondre » et ils déposent des textes de manière aléatoire sur la plateforme. Ceci empêche une bonne communication, puisque le partenaire doit chercher I'intervention de son binôme. Ainsi U6 écrit, pour un exercice où il est censé réagir au texte de son partenaire, en semaine 2: "je ne trouve pas le texte de mon jumeau/jumelle !!!". Chaque texte déposé aurait dû impérativement s'inscrire dans un déroulé de manière à enclencher de façon systématique le dialogue entre les pairs, et ce, dès le début du projet.

L'engagement des participants dans la télécollaboration se remarque par la rédaction de textes parfois longs. Souvent, celui qui répond a tendance à rédiger une réponse d'une longueur semblable à celle du texte initialement rédigé par le partenaire. Le dispositif ne comprenait aucune consigne en ce qui concerne la manière de communiquer avec les partenaires. L'analyse révèle aussi que, pour une des dyades ayant bien fonctionné (F2/U2), la participante Flinders invite dès la première interaction sa partenaire à la tutoyer, marquant ainsi sa volonté de rapprochement. Lors de l'activité qui consiste à écrire un texte à la manière de Houston, et à évoquer les émotions qui peuvent être présentes lors d'un passage entre le pays d'origine et le pays d'immigration, U2 fait part à sa partenaire australienne de plusieurs réflexions interculturelles. Elle lui explique les différences en matière d'organisation entre le Canada et l'Algérie visibles dès l'arrivée à l'aéroport et fait part du chômage et du manque de perspectives pour les diplômés algériens. Elle évoque également le fait que les Canadiens se plaignent des embouteillages, mais que ceux-ci, comparés à ceux de l'Algérie, n'en sont pas, selon elle.

\section{Productions autobiographiques}

Dans les productions écrites autobiographiques, les enseignantes-chercheuses observent une grande diversité de choix narratifs, et ce, pour les deux groupes. Il faut également noter les efforts en matière d'expression, même si les compétences linguistiques de certains apprenants ont pu entraver la bonne compréhension de leur écrit (on pense ici à U5).

Quatre apprenants du groupe Flinders (F2, F11, F10 et F7) ont fait le choix de retenir la forme "dictionnaire " suggérée par Sembar (2010) dans le premier texte étudié. Cependant, chaque apprenant a interprété cette idée de manière personnelle, en prenant par exemple l'alphabet en sens inverse (F11) ou en illustrant son texte de photographies et d'une carte géographique (F2). Plusieurs étudiants de Flinders ou de I'UQAM ont choisi la forme chronologique (F3, F4, U1, U4, U6, U7, U11) ou antéchronologique (U9).

Il est à noter que 4 étudiants de I'UQAM (U2, U3, U5 et U8) et une étudiante de Flinders (F5) ont choisi de rédiger un texte à la $3^{\mathrm{e}}$ personne du singulier, marquant ainsi une volonté de distanciation. Cette possibilité leur avait été offerte par l'enseignante. Pour les étudiants UQAM, le contenu des productions a été orienté sur leur adaptation au Québec ou sur les raisons qui les ont poussés à quitter leur pays d'origine. Dans leurs textes, les étudiantes de I'UQAM s'expriment souvent en tant que mères, et insistent sur le fait qu'offrir un avenir meilleur à leurs enfants est essentiel dans leur décision de migrer puis de rester au Québec. Le texte très émouvant d'une des étudiantes (U10) prend la forme d'une lettre qu'elle adresse à son fils à naître. 


\section{Échanges interculturels}

Les échanges écrits sur la plateforme ont mis en avant la pertinence qu'il y a à proposer une variété d'activités invitant les apprenants à parler d'eux-mêmes de manière directe ou bien à le faire de manière indirecte en partageant avec leur partenaire leur interprétation des textes lus. Ainsi, la rencontre interculturelle a constitué une partie importante du projet. Celui-ci, bien que limité dans le temps et ambitieux par ses objectifs, a donné l'opportunité aux apprenants de faire un retour sur leurs propres expériences interculturelles et d'en témoigner. II a aussi permis que s'établissent de véritables échanges interculturels, où chacun a mis en exergue des éléments cruciaux de sa culture ou encore a posé un regard sur les objets témoins de sa culture et sur ce qui, au-delà des objets, fait partie de la culture (F2).

Dans les questionnaires distribués au début et en fin de parcours, nous posions aux participants la question : "Qu'évoque pour vous l'expression "interculturel" ? À quoi le mot "interculturel" vous fait-il penser ?». On note, comme on peut s'y attendre dans les réponses des étudiants de I'UQAM, qui sont des personnes immigrantes, que celles-ci font souvent référence à leur environnement immédiat. La définition d'“interculturel” proposée par l'étudiant U8 nous semble particulièrement intéressante : "Ce sont les échanges et de délicatesse [sic] dans les relations entre cultures. Cette expression c'est la résolution des conflits ou encore la philosophie étudient [sic] les phénomènes résultant de la rencontre de plusieurs cultures, ou relations interculturelles ».

\section{Conclusion}

Les enseignantes-chercheuses ont comparé le dispositif proposé et les résultats saillants de la recherche conduite. Ces résultats montrent la pertinence d'une approche qui tisse des liens entre la lecture et l'écriture à partir de textes littéraires sur le thème de la migration et de l'autobiographie. L'analyse met également en évidence la qualité et l'intérêt des textes autobiographiques produits par les apprenants, si on les considère, entre autres, sous l'angle interculturel. II aurait d'ailleurs été pertinent d'avancer la date de soumission des textes individuels afin que ceux-ci puissent faire l'objet d'une discussion à l'intérieur des dyades avant la fin du projet. Enfin, on peut remarquer que le dispositif n'incluait pas de phase proprement consacrée à l'écriture collaborative. II pourrait être suggéré aux participants, lors d'une future reprise du projet, d'écrire des textes communs, suscitant ainsi davantage le dialogue interculturel. Somme toute, l'objectif principal du dispositif de télécollaboration a été atteint. Des étudiants universitaires de diverses langues et cultures ont réussi à participer à des échanges interculturels en ligne, en utilisant le français comme langue seconde ou étrangère. Ils ont pu partager des informations sur eux, sur leur langue et leur culture. Ces échanges ont permis d'aborder des thèmes interculturels comme la migration et l'autobiographie migratoire.

\section{Références bibliographiques}

Adami, H. (2008). L'acculturation linguistique des migrants: des tactiques d'apprentissage à une sociodidactique du français langue seconde. Dans C. Extramiana et J. Sibille (dir.), Migrations et plurilinguisme en France (p. 1015). Paris : Didier.

Akiyama, Y., et Cunningham, D. J. (2018). Synthesizing the Practice of SCMC-based Telecollaboration : A Scoping Review. CALICO Journal, 35(January), 49-76. https://doi.org/10.1558/cj.33156

Amireault, V. et Deraîche, M. (2015). Jumelage en ligne. Une expérience de communication interculturelle. Dans N. Carignan, M. Deraîche et M.-C. Guillot (dir.), Jumelages interculturels: communication, inclusion et intégration (p. 131-144). Montréal : Presses de l'Université du Québec.

Appel, C. et Mullen, T. (2000). Pedagogical considerations for a web-based tandem language learning environment. Computers and Education, 34(3-4), 291-308. https://doi.org/10.1016/S0360-1315(99)00051-2

Barbizet-Namer, L. (2009). Nouvelles odyssées. 50 auteurs racontent l'immigration. Paris : Cité Nationale de I’Histoire de I’Immigration.

Bélair, K. (2010). L'écriture migrante au Québec: L'interculturalisme dans le discours littéraire et politique. Montréal : Université McGill. https://doi.org/10.1017/CB09781107415324.004 
Cardell, K. et Douglas, K. (2016). Why literature students should practise life writing. Arts and Humanities in Higher Education, 1-18. https://doi.org/10.1177/1474022216635825

Carignan, N., Deraîche, M. et Guillot, M.-C. (2015). Jumelages interculturels. Communication, inclusion et intégration. Montréal : Presses de l'Université du Québec.

Chun, D. M. (2015). Language and culture learning in higher education via telecollaboration. Pedagogies, 10(1), 521. https://doi.org/10.1080/1554480X.2014.999775

Clifford, E. et Kalyanpur, M. (2011). Immigrant narratives : Power, difference, and representation in young-adult novels with immigrant protagonists. International Journal of Multicultural Education, 13(1), 1-20.

Conseil de l'Europe. (2014). Le mouvement contre la haine. Une campagne jeunesse du Conseil de l'Europe contre le discours de haine en ligne. Retrieved March 26, 2018, from https://www.nohatespeechmovement.org

Conseil de l'Europe. L'Autobiographie des rencontres interculturelle. https://www.coe.int/fr/web/autobiography-intercultural-encounters

Cziko, G. (2004). Electronic Tandem Language Learning (eTandem): A third approach to second language learning for the $21^{\text {st }}$ Century. CALICO Journal, 22(1), 25-39.

De Croix, S. et Ledur, D. (2014). Les visages du lecteur implicite dans la littérature migrante pour adolescents. Le Langage et l'Homme, 49(1), 177-185.

ECRI. (2016). Rapport de l'ECRI sur la France. Strasbourg. https://doi.org/10.3406/bmsap.1906.8173

Gagliardone, I., Gal, D., Alves, T. et Martinez, G. (2015). Countering online hate speech. Paris : Éditions de I'UNESCO. http://unesdoc.unesco.org/images/0023/002332/233231e.pdf

Helm, F. (2014). From the field. eLearning Papers, 38, 1-8. www.openeducationeuropa.eu/en/elearning_papers

Jobin, R. et Tamiozzo, J. (2016). Voix croisées. Montréal : Chenelière Éducation.

Kunigis, P. et Marcotte, V. (2014). Voix migrantes. Naître ailleurs, vivre ici. Montréal : Québec Amérique.

Lebrun, M. et Collès, L. (2007). La littérature migrante dans l'espace francophone. Belgique-France-Québec-Suisse: E.M.E.

Legendre, R. (2005). Dictionnaire actuel de l'éducation ( $3^{\mathrm{e}}$ édition). Montréal : Guérin.

Lejeune, P. (2001). Enseigner à écrire l'autobiographie. Dans M. H. Roques (dir.), L'autobiographie en classe (p. 1322). Midi-Pyrénées : Delagrave-CRDP.

Lewis, T. et O’Dowd, R. (2016). Onine intercultural exchange and foreign language learning. A systematic review. Dans R. O’Dowd et T. Lewis (dir.), Online intercultural exchange. Policy, pedagogy, practice (p. 21-66). New York/London : Routledge.

Maizonniaux, C. (2018). La littérature de jeunesse en classe de langue. Approche créative en contexte universitaire australien. Grenoble : Université Grenoble-Alpes Éditions

Maizonniaux, C., Deraîche, M. et Saffari, H. (2017). Télécollaboration et autobiographie : un projet croisé FLS/FLE. Les Langues Modernes, 2017(1), 59-64.

Molinié, M. (2011). La méthode biographique : de l'écoute de l'apprenant de langues à l'herméneutique du sujet plurilingue. Dans P. Blanchet et P. Chardenet (dir.), Guide pour la recherche en didactique des langues et des cultures. Approches contextualisées (p. 144-155). Paris : Éditions des archives contemporaines.

Muller Hartmann, A. (2000). The role of tasks in promoting intercultural learning in electronic learning networks. Language Learning and Technology, 4(2), 129-147.

Muller-Hartmann, A. (2007). Teacher role in telecollaboration: Setting up and managing exchanges. Dans R. O’Dowd (dir.), Online intercultural exchange. An Introduction for foreign language teachers (p. 167-191). Clevedon : Multilingual Matters. 
O'Dowd, R. (2006). Telecollaboration and the development of intercultural communicative competence. Berlin : Langenscheidt.

O'Dowd, R. (2018). From telecollaboration to virtual exchange: state-of-the-art and the role of UNICollaboration in moving forward. Journal of Virtual Exchange, 1(0), 1-23. https://journal.unicollaboration.org/article/view/877

O’Dowd, R. et Waire, P. (2009). Critical issues in telecollaborative task design. Computer Assisted Language Learning, 22(2), 173-188. https://doi.org/10.1080/09588220902778369

Ollivier, C. et Puren, L. (2014). Du bon usage du Web 2.0 ou comment faire rimer innovation technologique avec innovation pédagogique. Québec Français, 173, 61-63.

Patton, M. Q. (2002). Qualitative Research and Evaluative Methods (3 éd.). Thousand Oaks : Sage.

Reuter, Y. (1994). Problématique des interactions lecture-écriture. Dans Y. Reuter (dir.), Les interactions lectureécriture (p. 1-20). Berne : Perter Lang.

Roche, A. (2001). Soi-même comme un autre. Dans M. H. Roques (dir.), L'autobiographie en classe (p. 45-54). MidiPyrénées : Delagrave-CRDP.

Sembar, E. (2010). Le dictionnaire amoureux de la Palestine. Paris : Plon.

Unicollaboration. (2014). Position paper : Virtual exchange in the European higher education area. https://unicollaboration.eu/sites/default/files/Position paper_0.pdf

Van der Maren, J.-M. (2010). Les recherches qualitatives : des critères variés de qualité en fonction des types de recherche. Dans L. Paquay, M. Crahay et J.-M. De Ketele (dir.), L'analyse qualitative en éducation. Des pratiques de recherche aux critères de qualité. Hommage à Michael Huberman (2 éd., p. 69-84). Bruxelles : De Boeck Supérieur.

Vaatz Laaroussi, M., Armand, F., Kanouté, F., Rachedi, L., Steinbach, M., Stoica, A. et Rousseau, C. (2010). Écriture et histoires familliales de migration : une recherche action pour promouvoir les compétences à écrire des élèves allophones immigrants et réfugiés dans les écoles primaires et secondaires du Québec (rapport de recherche). Québec : Gouvernement du Québec, Fonds de recherche Société et culture. http://www.frqsc.gouv.qc.ca/documents/11326/449040/PT_VatzM_r\%C3\%A9sum\%C3\%A9\%202014_comp \%C3\%A9tence+\%C3\%A9criture+immig/5f164a3d-9a58-4151-a617-3c6fddd74423

\section{Annexe A. Textes de littérature migrante proposés aux apprenants}

Cheng, F. (2002). Le dialogue. Paris : Desclée de Brouwer. Lecture des pages 163-164.

Houston, N. et Sebbar, L. (1999). Autopsie de l'exil. Dans Lettres parisiennes. Paris : J'ai lu. Lecture des pages 117-118.

Lacoue-Labarthe, I. et Sanbar, E. (2013). De l'exil forcé à l'exil choisi... entretien avec Elias Sanbar. Diasporas, 22, 21-37.

Laferrière, D. (2012). Chronique de la dérive douce ( $2^{\mathrm{e}}$ éd.). Montréal : Les Éditions Boréales. Lecture des pages 205-209.

Schneider, A. (2013). La littérature de jeunesse migrante. Paris : L'Harmattan. Lecture des pages 17-19.

Thuy, K. (2010). Ru. Paris : Le Livre de Poche. Lecture des pages 11-19. 